\title{
Study on the Electrospun CNTs/Polyacrylonitrile-Based Nanofiber Composites
}

\author{
Bo Qiao, Xuejia Ding, Xiaoxiao Hou, and Sizhu Wu \\ Key Laboratory of Carbon Fiber and Functional Polymers, Ministry of Education, College of Materials Science \& Engineering, \\ Beijing University of Chemical Technology, Beijing 100029, China
}

Correspondence should be addressed to SizhuWu,wusz@mail.buct.edu.cn

Received 16 June 2011; Revised 21 July 2011; Accepted 3 August 2011

Academic Editor: Steve Acquah

Copyright ( 2011 Bo Qiao et al. This is an open access article distributed under the Creative Commons Attribution License, which permits unrestricted use, distribution, and reproduction in any medium, provided the original work is properly cited.

CNTs/PAN nanofibers were electrospun from PAN-based solution for the preparation of carbon nanofiber composites. The asspun polyacrylonitrile-based nanofibers were hot-stretched by weighing metal in a temperature controlled oven. Scanning electron microscopy (SEM) and transmission electron microscopy (TEM) were used to characterize the morphology of the nanofibers, which indicated that carbon nanotubes were dispersed well in the composites and were completely wrapped by PAN matrix. Because of the strong interfacial interaction between CNTs and PAN, the CNTs/PAN application performance will be enhanced correspondingly, such as the mechanical properties and the electrical conductivity. It was concluded that the hot-stretched $\mathrm{CNTs} / \mathrm{PAN}$ nanofibers can be used as a potential precursor to produce high-performance carbon composites.

\section{Introduction}

Electrospinning provides a straightforward and cost-effective approach to produce fibers from polymer solutions or melts having the diameters ranging from submicrons to nanometers [1-4]. Various polymers have been successfully electrospun into ultrafine fibers in recent years mostly in solvent solution and some in melt form. Potential applications based on such fibers specifically used as reinforcement in nanocomposites have been realized [5]. PAN is the most widely used precursor for manufacturing high-performance fibers due to its combination of tensile and compressive properties as well as the high carbon yield [6]. Conventional PAN-based carbon fibers typically have diameters ranging from 5 to $10 \mathrm{um}$ [7]. However, the electrospun PAN nanofibers are uniform with the diameters of approximately $300 \mathrm{~nm}[8,9]$, which is more than 30 times smaller than their conventional counterparts. The high specific surface area of electrospun polymer and carbon nanofibers leads to the enhanced properties in various applications such as electrodes in fuel cells and supercapacitors. In spite of significant improvements in specific surface area of the PAN nanofibers, several drawbacks of polymer nanofibers are still present. For instance, the electrical conductivity of PAN is an order of $\mu \mathrm{S} / \mathrm{cm}$. The microstructures and the related mechanical and/or electrical properties of the electrospun carbon nanofibers are still not clear.

Carbon nanotubes (CNTs) possess several unique mechanical, electronic, and other kinds of characteristics. For instance, single carbon nanotube has a modulus as high as several thousands of GPa and a tensile strength of several tens of GPa [10]. It is found that reinforcement of polymers by CNTs may significantly improve their mechanical properties, thermal stability, electric conductivity, and other functional properties [11]. It has been shown that significant interactions exist between PAN chains and CNTs, which lead to higher orientation of PAN chains during the heating process [12]. These outstanding properties make the polymer nanofibers optimal candidates for many important applications. It is also noted that single-wall carbon nanotube(SWNT-) reinforced polyimide composite in the form of nanofibrous film was made by electrospinning to explore a potential application for spacecrafts [13]. Carbon nanofibers for composite applications can also be manufactured from precursor polymer nanofibers [14]. Such kind of continuous carbon nanofiber composite also has potential applications as filters for separation of small particles from gas or liquid, supports for high temperature catalysts, heat management 


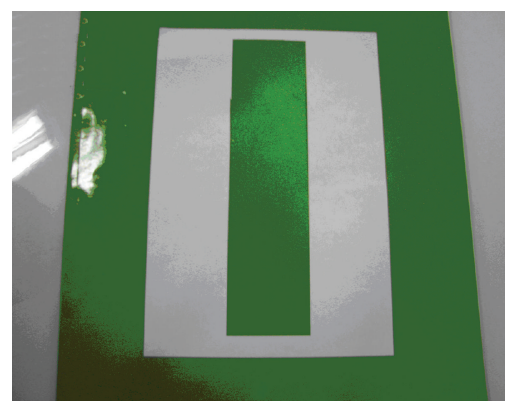

(a)

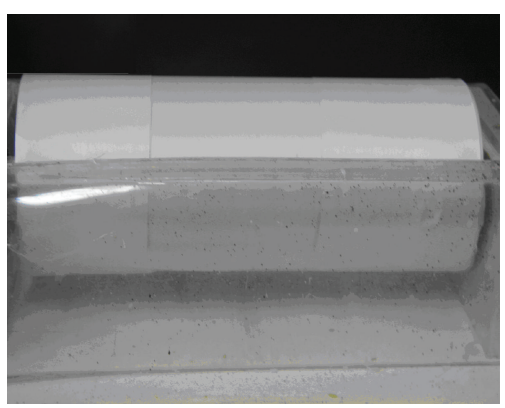

(b)

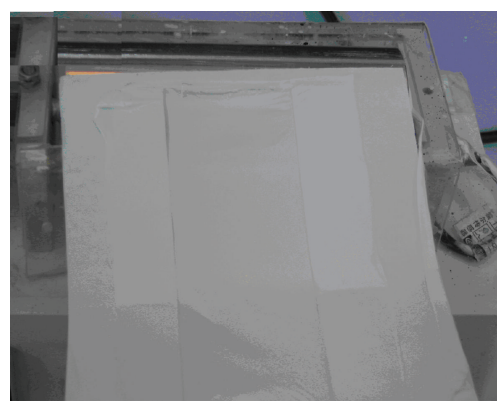

(c)

FIgURE 1: Schematic diagram of the frame to prepare the aligned nanofibers films. (a) The $16 \mathrm{~cm} \times 12 \mathrm{~cm}$ paper frame with the hollow of $16 \mathrm{~cm} \times 4 \mathrm{~cm}(\mathrm{~b})$ on the rotating $\operatorname{drum}(\mathrm{c})$ after electrospinning.

materials in aircraft, and semiconductor devices, as well as promising candidates as small electronic devices, rechargeable batteries, and supercapacitors [15]. Fibrous materials used for filter media provide advantages of high filtration effciency and low air resistance [16].

However, before full realization of their high performance, the following two crucial issues have to be solved: (i) dispersion and orientation of CNTs in the nanofiber $[17,18]$, good interfacial bonding is required to achieve load transfer across the CNT smatrix interface [19]; (ii) the macroscopic alignment in the nanofibers [20] and the orientation and crystallinity of polymer chains. Therefore, the manufacturing process and characterization methods for the microstructures and mechanical properties of PAN and PAN-based nanofibers have been studied in this paper.

\section{Experimental}

2.1. Materials. PAN used in this study included PAN/methyl acrylate/itaconic acid (93:5.3:1.7 w/w) (average molecular weight of $100000 \mathrm{~g} / \mathrm{moL}$ ) which was purchased from UK Courtaulds Ltd. Since N,N-dimethylformamide (DMF) is the common solvent of PAN $[3,5,8,13,14]$ which can easily evaporate during the electrospinning, so in this study the DMF was selected as solvent. It was purchased from Beijing Chemical Plant Co. To uniformly disperse the CNTs in the organic polymer matrix, the CNTs are modified to form an individually polymer-wrapped structure [17]. These effects caused the wrapped nanotubes to be much more readily suspended in concentrated SWNTs solutions and suspensions, which in turn substantially enabled manipulation of SWNTs into various bulk materials, including films, fibers, solids, and composites $[21,22]$.

\subsection{Formation of Electrospun PAN/SWNTs Nanofiber Com-} posites. The PAN and PAN-based nanofibers can be made by electrospinning with the nominal electric field on the order of $1 \mathrm{kV} / \mathrm{cm}$. In the electrospun process, the PAN or $\mathrm{CNTs} / \mathrm{PAN}$ solution is held by its surface tension at the end of a capillary, such as a stainless steel needle. The voltage between the electrode and the counter electrode could be controlled by the high voltage power supply such as setting at $14-16 \mathrm{kV}$. The collector rotated at $6.6 \mathrm{~m} / \mathrm{s}$ surface speed,

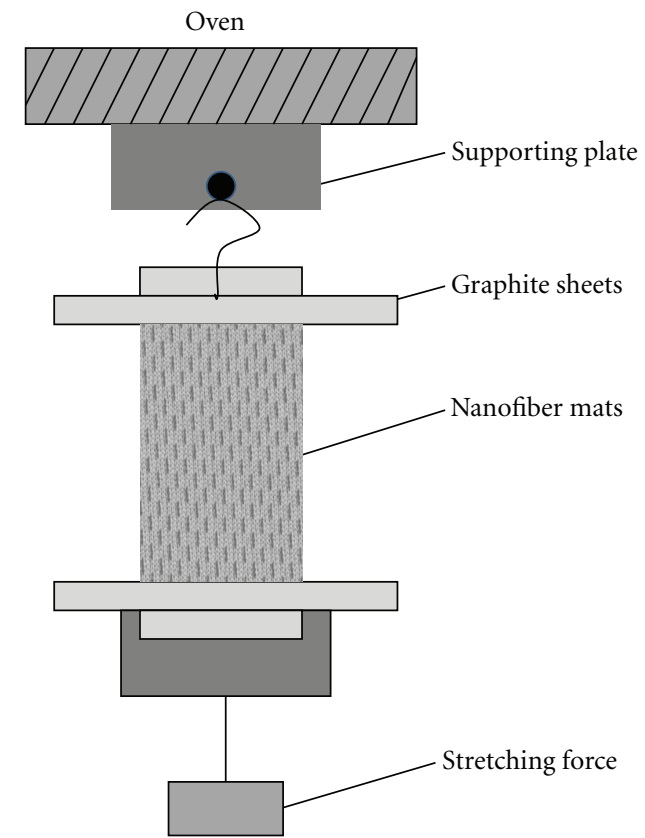

FIgURE 2: The schematic representation of the experimental setup for hot-stretching process.

by which the high speed rotating collector could align the nanofibers into the nanofiber sheets.

The relatively aligned PAN nanofibers and PAN/SWNTs composite nanofibers can be obtained by electrospining with the set-up of a rotating instrument. Thus, for collection of the large area aligned nanofibers, a parallel rotating drum can be adopted. Such as in our study, the $0.16 \mathrm{~m}$ perimeter collector rotated at a surface speed about $6.6 \mathrm{~m} / \mathrm{s}$, that the high speed rotating collector could align the nanofibers into the nanofiber sheets. Figure 1 showed the schematic diagram of the frame to prepare the aligned nanofibers films with (a) the $16 \mathrm{~cm} \times 12 \mathrm{~cm}$ paper frame with the hollow of $16 \mathrm{~cm} \times$ $4 \mathrm{~cm}$ wrapping around (b) the rotating drum and (c) the sheet after electrospinning. And the SEM photographs of different speeds of rotation were shown in Figure 2, which that indicated the higher rotating speed leads to higher alignment of the nanofibers. 
The SWNTs-based PAN composite solution was prepared as follows: (1) first, a given weight of SWNTs was first dispersed for $2 \mathrm{~h}$ in DMF through mild bath sonication, which was followed by the addition of PAN (128.91 mg per milliliter of SWNTs/DMF solution); (2) then, the mixture was mechanically stirred overnight at $40^{\circ} \mathrm{C}$ using a magnetic stirrer to yield a homogeneous solution.

During the electrospinning process, however, the whirlpool jet from the pinhead to the collector still made it difficult to get the unidirectional alignment in a large-area sheet [23] and the subsequent hot stretched procedure is particularly useful and is also the key process during the manufacturing of carbon fibers. Also, the electrospun nanofibers needed a subsequent hot-stretch to improve the fiber alignment. The PAN nanofibers and PAN/SWNTs composite nanofibers can be hot-stretched according to the method proposed by Johnson et al. [11, 12]. Both ends of the nanofiber sheet (size of $4 \mathrm{~cm}$ width $\times 10 \mathrm{~cm}$ length with $17 \mu \mathrm{m}$ thickness) can be clamped with the pieces of graphite plates. Then, one end was fixed to the ceiling of the oven and the other end can be weighted by some of metal poise $(75 \mathrm{~g})$ to give a desired tension and elongation in the temperature-controlled oven at $135 \pm 2^{\circ} \mathrm{C}$ for $5 \mathrm{~min}$. The schematic diagram of hot stretching of nanofiber sheet is shown in Figure 2. The stretching ratio, $\lambda$, can be calculated from $\lambda=L / L_{0}$, where $L$ and $L_{0}$ are the lengths of nanofiber sheet after and before the hot stretching, respectively.

2.3. Characterization. Morphological and structural examinations of the CNTs/PAN nanofibers were performed using scanning electron microscopy (SEM, HITACHI S4700 FEG-SEM) and the transmission electron microscopy (transmission electron microscopy (TEM, HITACHIH-800). The glass transition temperature $T_{g}$ of the PAN nanofiber and PAN/SWNTs nanofiber were examined using differential scanning calorimetry (DSC, METTLER-TOLEDO STARe system). The samples were heated at a scanning rate of $20^{\circ} \mathrm{C} / \mathrm{min}$ under nitrogen atmosphere in order to diminish oxidation. The value of $T_{g}$ was found by differentiating the heat flow curve with the temperature. Mechanical test was performed by using an LR30K Electromechanical Universal Testing Machine (LLOYD Company). There were eight specimens used for each nanofiber sample where the samples were prepared in $5 \mathrm{~mm}$ width and $20 \mathrm{~mm}$ length. The tensile speed was $20 \mathrm{~mm} / \mathrm{min}$. Electrical conductivities of electrospun PAN/SWNTs nanofiber composites were measured using a ZC43 ultrahigh resistance measuring machine (Shanghai Meter Plant Co., Ltd.) at room temperature and ambient condition.

\section{Results and Discussion}

3.1. Morphology and Microstructures of the Electrospun Composites Nanofibers. It can be seen from Figure 3 that there is no obvious conglutination in the nanofibers after the introduction of SWNTs, which proved that SWNTs were relatively dispersed well in the composites. And Figure 3(c) showed the hot stretched PAN nanofibers, documenting the better alignment along the sheet axis after the hot stretched process. It can further be found that the alignment of the fibers became closer to parallel after being hot stretched. Also, the average diameters of the original as-spun fibers were significantly reduced from $200 \mathrm{~nm}$ to $120 \mathrm{~nm}$ after hot stretching.

Figure 4 showed the SEM micrographs of PAN/SWNTs nanofibers with different SWNTs concentrations. The pure PAN nanofibers in Figure 4(a) were straight with a smooth surface and an average diameter of about $200 \mathrm{~nm}$. The nanofibers became straighter, and the average diameter was increased with increasing SWNT concentrations. For instance, in Figure 4(b) the average diameter was about $300 \mathrm{~nm}$. It was noted that SWNTs embedded in PAN nanofiber were mostly aligned along the nanofiber axis. When the concentration of the SWNTs increased to $1 \mathrm{wt} \%$, the surface of the composite fibers became a litter rough and the average diameter was a little bit smaller than the one of $0.5 \%$ wt SWNTs/PAN, which indicated that at high concentration some SWNTs might not be completely embedded into the nanofiber matrix [18].

In order to demonstrate that the prepared nanofibers do contain some oriented SWNTs, transmission electron microscopy (TEM) can be utilized to view the alignment and orientation of SWNTs within the nanofibers produced. Since the SWNTs possessed a high electron density compared with the PAN polymer matrix, SWNTs appeared as darker tubular structures embedded in the PAN/SWNTs composite nanofibers. It can be seen that the SWNTs are completely wrapped by the PAN matrix. TEM images revealed that in some regions nanotubes oriented well along the fiber axis but the nanotube distribution (number and orientation of the tubes) within a fiber may vary quite significantly (Figures $5(\mathrm{~b}), 5(\mathrm{c})$, and $5(\mathrm{~d}))$.

The temperature at which the transition in the amorphous regions between the glassy and rubbery state occurs is called the glass transition temperature, which is related directly to the segment movement of polymer chain. The $T_{g}$ of the PAN nanofiber and PAN/SWNTs nanofiber can be examined using differential scanning calorimetry (DSC). Figure 6(a) showed the DSC curves of PAN and PAN/SWNTs nanofibers. It can be seen that the $T_{g}$ is increased by about $3^{\circ} \mathrm{C}$ by incorporating $0.75 \mathrm{wt} \%$ SWNTs into the PAN matrix. The improvement in the $T_{g}$ stemmed from a stronger interfacial interaction and possible covalent bonding between PAN and the SWNTs. Figure 6(b) showed the DSC thermograms where the peak of PAN/SWNTs was higher than the one of pure PAN nanofibers. All these results suggested that the mobility of PAN chains is reduced due to the constraint effect of SWNTs [5].

\subsection{Application Performance of the Electrospun Composites} Nanofibers. One of the most important applications of engineering fibers such as carbon, glass, and Kevlar fibers was to be used as reinforcements in composites, which required that reinforced nanofibers should have a better mechanical properties [24]. Figure 7 showed the stress-strain curves of the PAN nanofibers and PAN/SWNTs nanofiber composites 


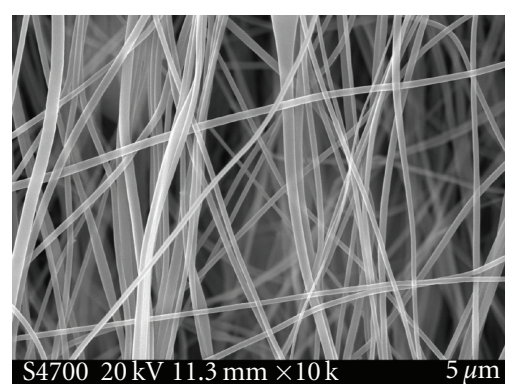

(a)

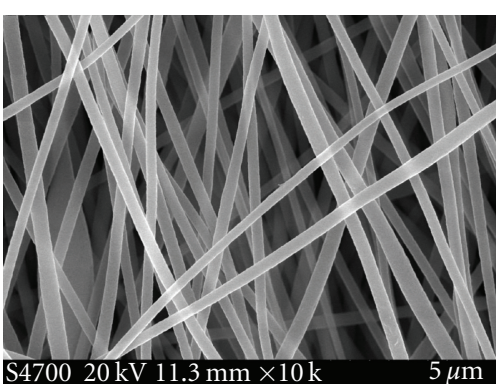

(b)

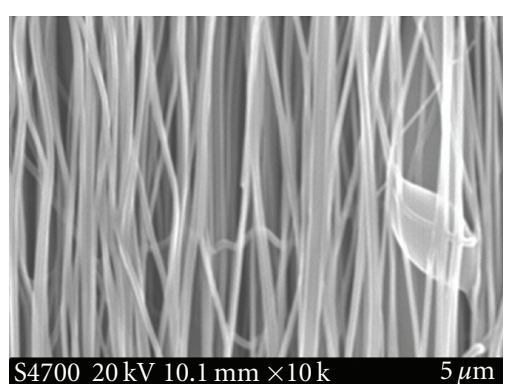

(c)

FIGURE 3: SEM micrographs: (a) as-spun pure partially aligned PAN nanofibers; (b) PAN/SWNTs composite nanofibers with SWNTs concentration $1 \mathrm{wt} \%$; (c) hot-stretched pure PAN nanofibers.

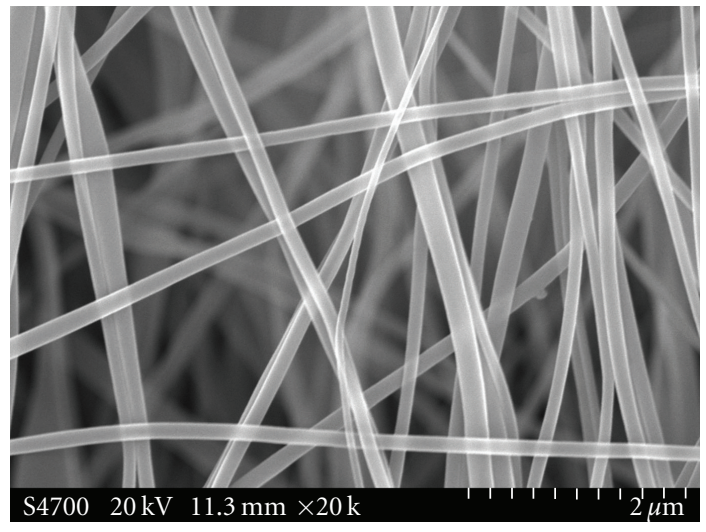

(a)

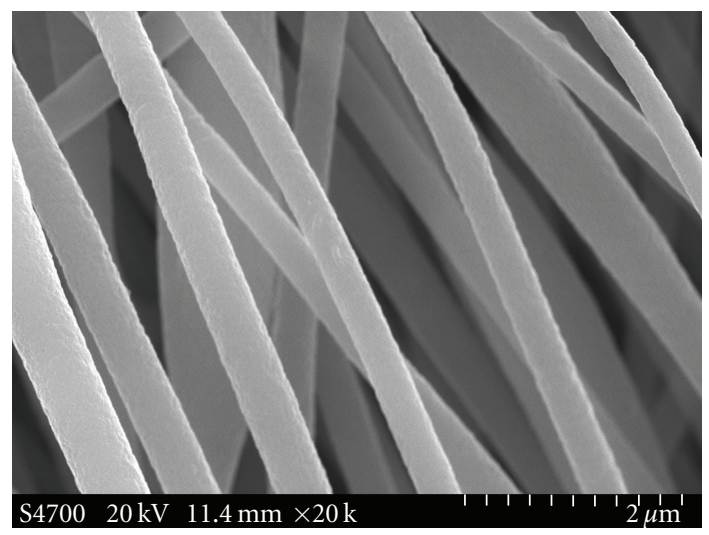

(c)

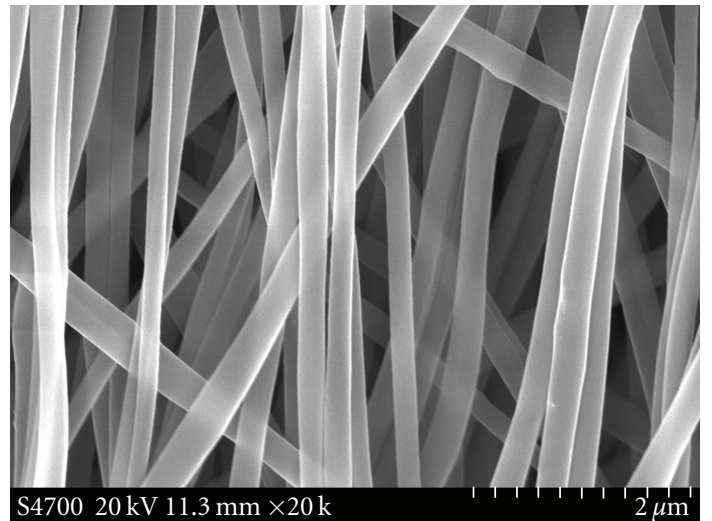

(b)

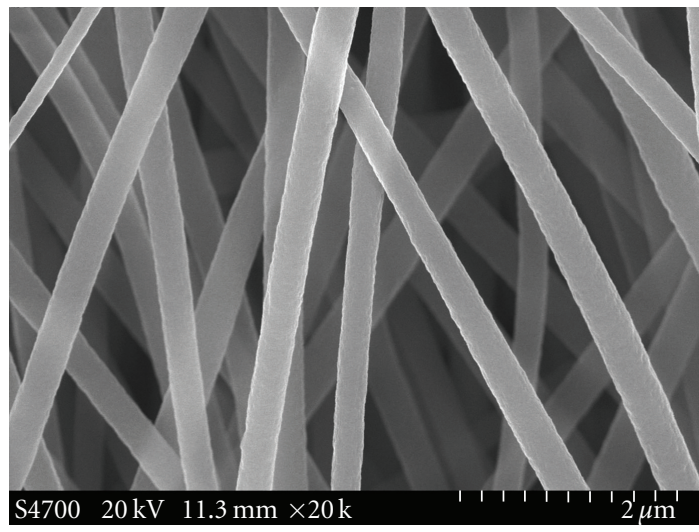

(d)

FIGURE 4: SEM micrographs of PAN/SWNTs nanofibers with different SWNTs concentration: (a) 0 wt $\%$, (b) 0.25 wt $\%$, (c) 0.5 wt $\%$, (d) $1 \mathrm{wt} \%$.

with different concentrations (hot stretching). It concluded that the introduction of SWNTs improves the modulus and tensile strength of the nanofiber. The tensile strength $128.76 \mathrm{MPa}$ of the nanocomposites at about $0.75 \%$ SWNTs by weight was increased with $58.9 \%$. Also the tensile modulus showed a peak value of $4.62 \mathrm{GPa}$ with $66.8 \%$ improvement. The (e) curve in Figure 7 deviated from the trend, which might be the reason of non uniform dispersion of SWNTs in high concentration. The significant improvement in strength and modulus was likely related to the good dispersion and orientation of SWNTs within the polymer matrix and the strong interfacial adhesion due to the SWNTs surface modification [5]. It can be concluded that both hot stretching and the introduction of SWNTs can improve the mechanical 


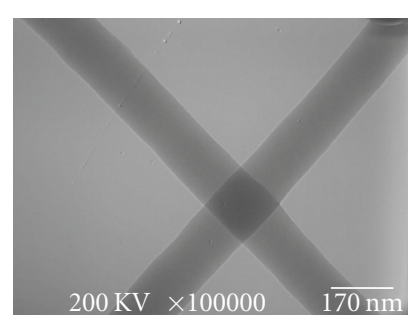

(a)

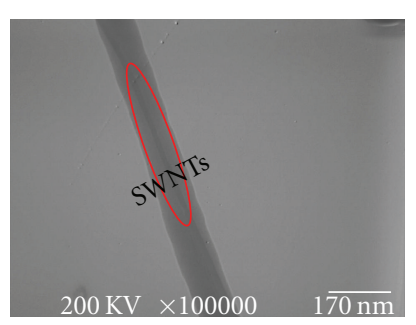

(b)

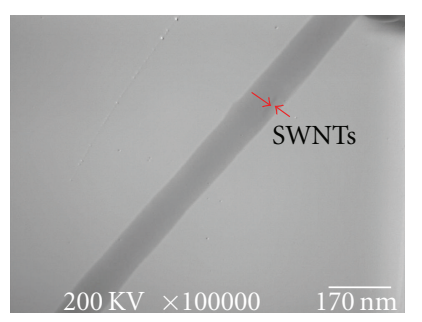

(c)

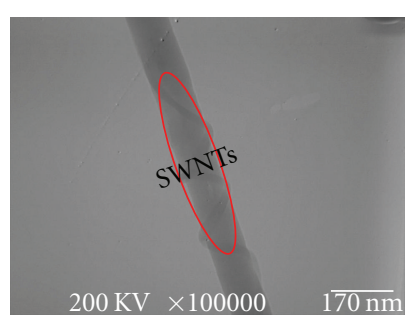

(d)

Figure 5: TEM images: (a) PAN nanofibers; (b)-(d) PAN/SWNTs nanofibers with SWNTs concentration 1 wt $\%$.

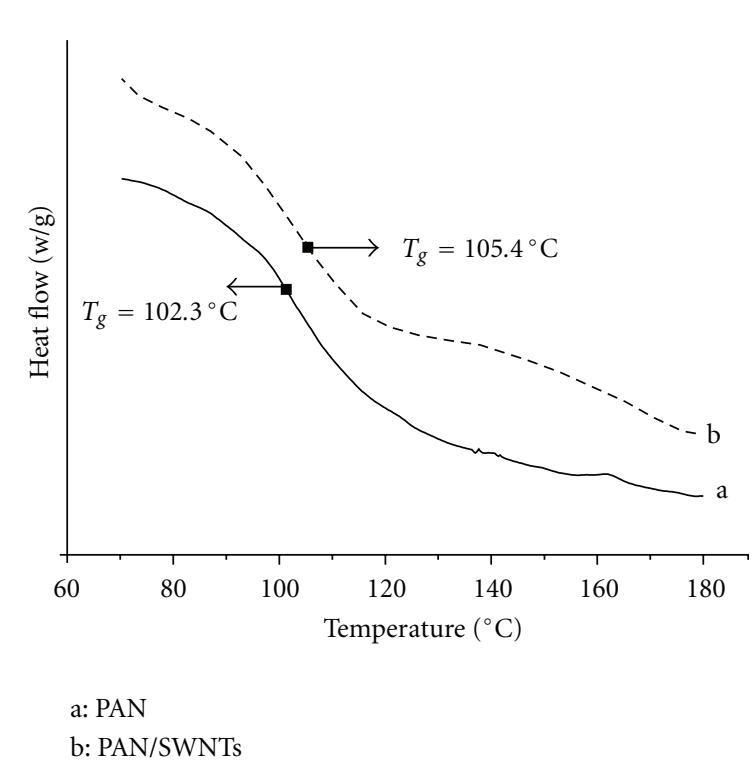

(a)

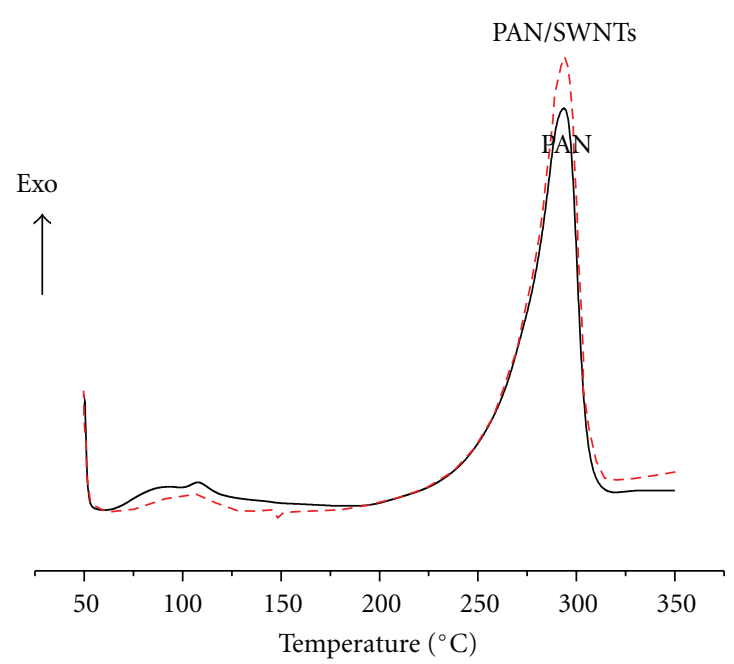

(b)

FIgURe 6: (a) $T_{g}$ from DSC of electrospun nanofibers: a: PAN nanofibers; b: PAN/SWNTs composite nanofibers; (b) DSC thermograms of electrospun PAN and PAN/SWNTs nanofibers.

properties of PAN-based nanofibers significantly. With these reinforcements, the composite materials can provide superior structure properties such as high modulus and strength to weight ratios.

High electrical conductivity was always desired to have high capacitance and high power density in supercapacitors [25]. Conductive nanofibers were expected to be used in the fabrication of tiny electronic devices or machines such as sensors and actuators. The electrical conductivities of electrospun PAN/SWNTs nanofiber composites can also be measured using the ultrahigh resistance measuring machine at room temperature and ambient condition. The electrical conductivities of this nanofiber composites can be obtained according to the following [20]:

$$
\rho_{v}=R_{v} \times \frac{21.23}{t},
$$

where $\rho_{v}$ was volume resistivity, $R_{v}$ was resistance, and $t$ was the thickness of the nanofiber films, respectively.
The electrical conductivity of the pure PAN nanofiber

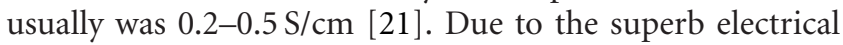
properties of SWNTs, a better electrical conductivity in PAN/SWNTs nanofiber composites was expected. There was no much change of the electrical conductivity in the SWNTs concentrations from 0 to $0.5 \%$, but the electrical conductivity of $0.75 \%$ concentration was detected suddenly up to $2.5 \mathrm{~S} / \mathrm{cm}$. This was because a good electrical conductivity required the percolating network be formed by the SWNTs. Therefore, it can be concluded that the percolating network in composite nanofibers will be formed at the concentration of 0.75 wt. $\%$ SWNTs.

\section{Conclusions}

In summary, this study showed that polyacrylonitrile-based carbon nanofibers, embedded with wrapped carbon nanotubes, can be obtained by electrospinning process. The composites exhibited improvements in thermal, tensile properties, and so forth. The CNTs/PAN nanofiber sheets with 


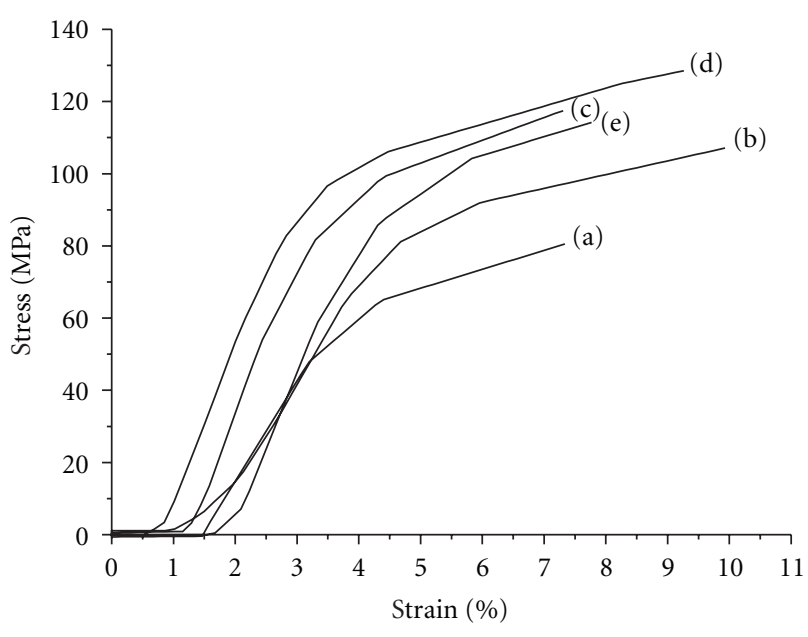

FIgURE 7: Stress-strain curves for PAN and PAN/SWNTs nanofiber: (a) pure PAN; (b) $0.25 \%$ SWNTs; (c) $0.5 \%$ SWNTs; (d) $0.75 \%$ SWNTs; (e) $1 \%$ SWNTs.

better alignments can be achieved by hot-stretched process. The morphology of the nanofibers characterized by SEM and TEM showed that carbon nanotubes were completely wrapped by the PAN matrix and oriented well along the fiber axis. Differential scanning calorimetry showed that the glass transition temperature of PAN increased by addition of SWNTs, indicating a strong interfacial interaction between PAN and SWNTs. Compared to pure PAN nanofibers, the mechanical property of the CNTs/PAN nanofibers exhibited quite improvement. For example, the tensile strength of the nanocomposites with $0.75 \%$ SWNTs by weight was increased with $58.9 \%$. Incorporation of SWNTs into the nanofibers also increases the electrical conductivity to $2.5 \mathrm{~S} / \mathrm{cm}$ for PAN/0.75\% SWNTs nanofiber composites. Thus, the composite nanofibers with the component of SWNTs can be used as the potential precursor to produce high-performance carbon nanofibers.

\section{Acknowledgment}

The authors gratefully acknowledge the financial support sponsored by the NSF of China (50973007).

\section{References}

[1] R. Andrews, D. Jacques, A. M. Rao et al., "Nanotube composite carbon fibers," Applied Physics Letters, vol. 75, no. 9, pp. 13291331, 1999.

[2] R. H. Baughman, A. A. Zakhidov, and W. A. De Heer, "Carbon nanotubes-the route toward applications," Science, vol. 297, no. 5582, pp. 787-792, 2002.

[3] Z. Bashir, S. P. Church, and D. Waldron, "Interaction of water and hydrated crystallization in water-plasticized polyacrylonitrile films," Polymer, vol. 35, no. 5, pp. 967-976, 1994.

[4] Y. V. Basova, D. D. Edie, Y. S. Lee, L. K. Reid, and S. K. Ryu, "Effect of precursor composition on the activation of pitchbased carbon fibers," Carbon, vol. 42, no. 3, pp. 485-495, 2004.
[5] W. J. Chou, C. C. Wang, and C. Y. Chen, "Characteristics of polyimide-based nanocomposites containing plasmamodified multi-walled carbon nanotubes," Composites Science and Technology, vol. 68, no. 10-11, pp. 2208-2213, 2008.

[6] I. Chun, D. H. Reneker, H. Fong et al., "Carbon nanofibers from polyacrylonitrile and mesophase pitch," Journal of Advanced Materials, vol. 31, no. 1, pp. 36-41, 1999.

[7] P. G. Collins, M. S. Arnold, and P. Avouris, "Engineering carbon nanotubes and nanotube circuits using electrical breakdown," Science, vol. 292, no. 5517, pp. 706-709, 2001.

[8] J. B. Donnet, T. K. Wang, J. C. Peng, and S. Rebouillat, Carbon Fibers, Marcel Dekker, New York, NY, USA, 1998.

[9] Y. Dzenis, "Spinning continuous fibers for nanotechnology," Science, vol. 304, no. 5679, pp. 1917-1919, 2004.

[10] A. Javey, J. Guo, Q. Wang, M. Lundstrom, and H. Dai, "Ballistic carbon nanotube field-effect transistors," Nature, vol. 424, no. 6949, pp. 654-657, 2003.

[11] J. Johnson, L. N. Phillips, and W. Watt, "The production of carbon fibers," British Patent, no. 1,110,790, 1965.

[12] J. Johnson, W. Watt, L. N. Phillips, and R. Moreton, "Improvements in or relating to carbonisable fibre and carbon fibre and their production,” British Patent, no. 1,166,251, 1966.

[13] A. Koganemaru, Y. Z. Bin, and M. Matsuo, "Composites of polyacrylonitrile and mutiwalled carbon nanotubes prepared by gelation/crystallization from solution," Advanced Functional Materials, vol. 14, pp. 842-850, 2004.

[14] D. Li, Y. Wang, and Y. Xia, "Electrospinning of polymeric and ceramic nanofibers as uniaxially aligned arrays," Nano Letters, vol. 3, no. 8, pp. 1167-1171, 2003.

[15] A. G. MacDiarmid, W. E. Jones, I. D. Norris et al., "Electrostatically-generated nanofibers of electronic polymers," Synthetic Metals, vol. 119, no. 1-3, pp. 27-30, 2001.

[16] P. Miaudet, C. Bartholome, A. Derré et al., "Thermo-electrical properties of PVA-nanotube composite fibers," Polymer, vol. 48, no. 14, pp. 4068-4074, 2007.

[17] E. R. Waclawik, J. M. Bell, R. G. S. Goh, A. Musumeci, and N. Motta, "Self-organization in composites of poly(3-hexylthiophene) and single-walled carbon nanotubes designed for use in photovoltaic applications," in BioMEMS and Nanotechnology II, vol. 6036 of Proceedings of the SPIE, Brisbane, Australia, December 2005.

[18] R. E. Smalley, D. T. Colbert, K. A. Smith, and O. Michael, "Polymer-wrapped single wall carbon nanotubes," US Patent, no. 201,001,437,18A, 2007.

[19] Z. M. Huang, Y. Z. Zhang, M. Kotaki, and S. Ramakrishna, "A review on polymer nanofibers by electrospinning and their applications in nanocomposites," Composites Science and Technology, vol. 63, no. 15, pp. 2223-2253, 2003.

[20] N. W. H. Cheetham and L. Tao, "Variation in crystalline type with amylose content in maize starch granules: an X-ray powder diffraction study," Carbohydrate Polymers, vol. 36, no. 4, pp. 277-284, 1998.

[21] D. H. Reneker and I. Chun, "Nanometre diameter fibres of polymer, produced by electrospinning," Nanotechnology, vol. 7, no. 3, pp. 216-223, 1996.

[22] S. D. McCullen, D. R. Stevens, W. A. Roberts, S. S. Ojha, L. I. Clarke, and R. E. Gorga, "Morphological, electrical, and mechanical characterization of electrospun nanofiber mats containing multiwalled carbon nanotubes," Macromolecules, vol. 40, no. 4, pp. 997-1003, 2007.

[23] E. P. S. Tan and C. T. Lim, "Mechanical characterization of nanofibers-a review," Composites Science and Technology, vol. 66, no. 9, pp. 1102-1111, 2006. 
[24] H. G. Chae, T. V. Sreekumar, T. Uchida, and S. Kumar, "A comparison of reinforcement efficiency of various types of carbon nanotubes in polyacrylonitrile fiber," Polymer, vol. 46, no. 24, pp. 10925-10935, 2005.

[25] I. H. Chen, C. C. Wang, and C. Y. Chen, "Fabrication and structural characterization of polyacrylonitrile and carbon nanofibers containing plasma-modified carbon nanotubes by electrospinning," Journal of Physical Chemistry C, vol. 114, no. 32, pp. 13532-13539, 2010. 

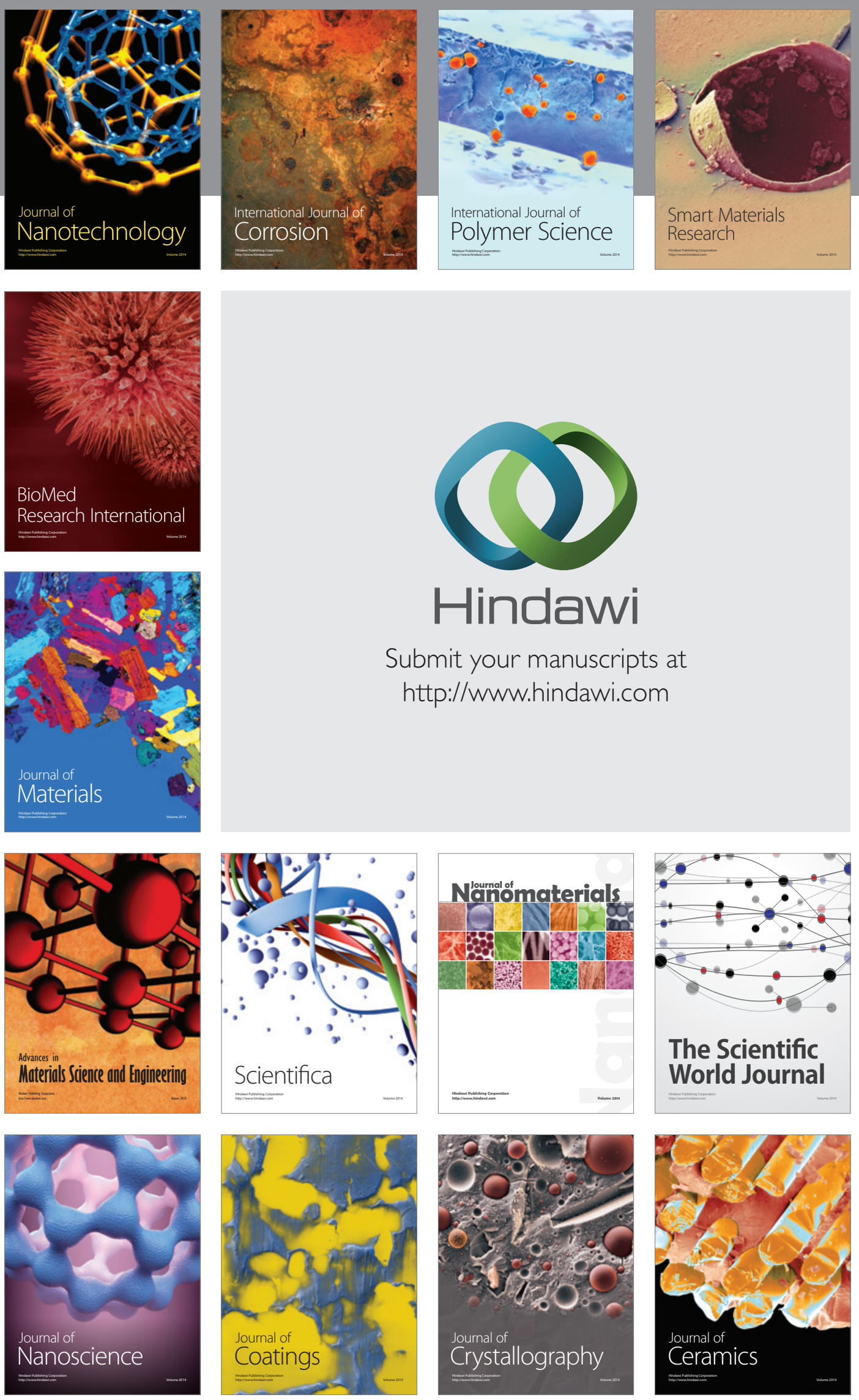

The Scientific World Journal

Submit your manuscripts at

http://www.hindawi.com

\section{World Journal}

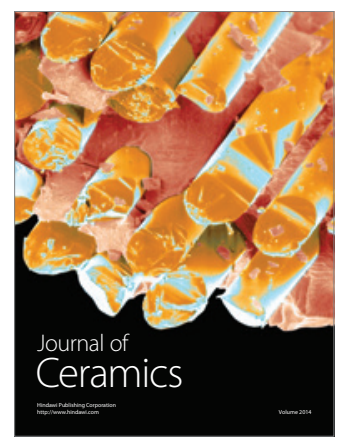

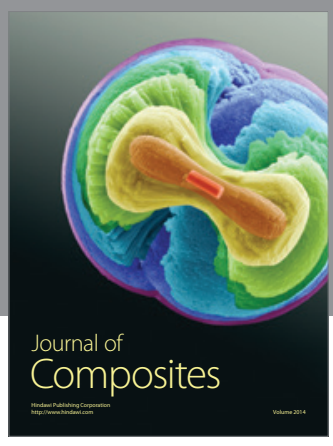
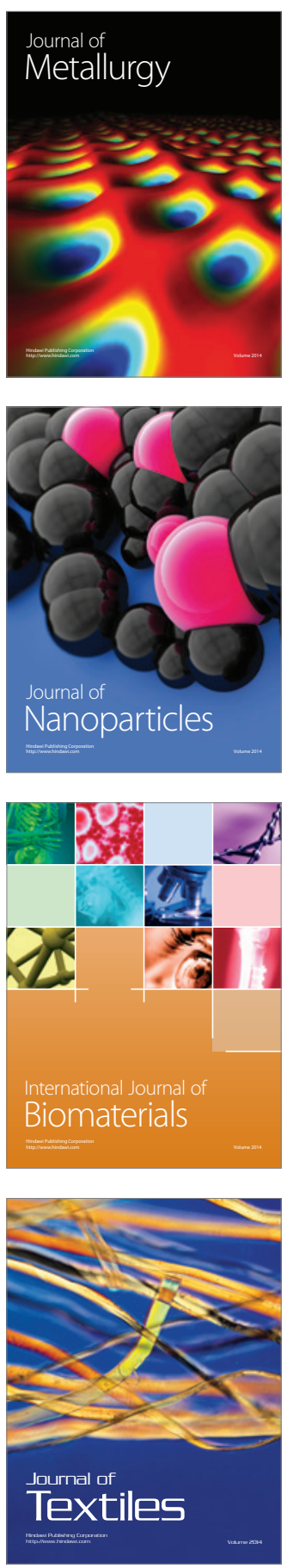